\title{
Anti-CEA/Anti-DTPA-In (F6-734) Bispecific Antibody
}

National Cancer Institute

\section{Source}

National Cancer Institute. Anti-CEA/Anti-DTPA-In(F6-734) Bispecific Antibody. NCI

Thesaurus. Code C68927.

A bispecific monoclonal antibody (BsMAb) consisting of the Fab fragment of an anti-CEA monoclonal antibody (F6) coupled to the Fab fragment of an anti-DTPA-In monoclonal antibody (734) with potential radioimmunotherapeutic activity. In a two-step pretargeted radioimmunotherapeutic approach, this BsMAb, localizing to CEA-expressing tumor cells via the F6 Fab frag ment, is introduced into patient first, followed by injection of indium 131-radiolabeled DTPA, which is recognized by the 734 Fab fragment of the BsMAb. Accordingly, a potentially lethal dose of indium 131 is delivered specifically to CEAexpressing tumor cells while minimizing radiotoxicity to normal tissues. CEA (carcinoembryonic antigen) is a tumor antigen overexpressed in many cancer types, including gastrointestinal, breast, non-small cell lung, and thyroid cancers. DTPA (diethylenetriaminepentaacetic acid) is a bivalent hapten. 